\title{
INTEGRALS OF CERTAIN UNIVALENT FUNCTIONS
}

\author{
RAM SINGH AND SUNDER SINGH
}

Abstract. In this paper we study integrals of certain univalent functions in the unit disc $E=\{z:|z|<1\}$ and extend some well-known results of Libera.

Introduction. Let $A$ denote the class of functions

$$
f(z)=z+\sum_{n=2}^{\infty} a_{n} z^{n}
$$

which are regular in the unit disc $E=\{z:|z|<1\}$. We denote by $S$ the subclass of univalent functions in $A$ and by $C, S^{*}$ and $K$ the subclasses of $S$ whose members are close-to-convex, starlike (with respect to the origin) and convex in $E$, respectively. A function $f \in A$ is said to be starlike of order $\alpha$, $\alpha<1$, in $E$ if and only if

$$
\operatorname{Re} \frac{z f^{\prime}(z)}{f(z)}>\alpha, \quad z \in E
$$

Similarly, $f \in A$ is said to be convex of order $\alpha, \alpha<1$, in $E$ if and only if

$$
\operatorname{Re}\left\{1+\frac{z f^{\prime \prime}(z)}{f^{\prime}(z)}\right\}>\alpha, \quad z \in E .
$$

We shall denote by $S^{*}(\alpha)$ and $K(\alpha)$ the subclasses of $A$ whose members satisfy (1) and (2), respectively. It is known that for $0<\alpha<1, S^{*}(\alpha) \subset S^{*}$, $K(\alpha) \subset K$ and that $S^{*}(0) \equiv S^{*}, K(0) \equiv K$.

Following Ruscheweyh [3] we denote by $K_{n}, n \in N_{0}=\{0,1, \ldots\}$, the subclass of $A$ whose members satisfy the condition

$$
\operatorname{Re}\left\{\frac{\left(z^{n} f(z)\right)^{(n+1)}}{\left(z^{n-1} f(z)\right)^{(n)}}\right\}>\frac{n+1}{2}, \quad z \in E .
$$

It is readily seen that $K_{0} \equiv S^{*}(1 / 2)$ and $K_{1} \equiv K$.

As observed by Ruscheweyh, $f \in K_{n}$ if and only if

$$
\operatorname{Re}\left\{\frac{D^{n+1} f(z)}{D^{n} f(z)}\right\}>\frac{1}{2} \quad(z \in E),
$$

Received by the editors June 13, 1978.

AMS (MOS) subject classifications (1970). Primary 30A32, 30A34.

Key words and phrases. Univalent, starlike and convex functions, convolution/Hadamard product. 
where

$$
D^{n} f(z)=\frac{z}{(1-z)^{n+1}} * f(z)
$$

(Here '*' stands for the Hadamard product/convolution of two regular functions.)

In [3] Ruscheweyh proved that for each $n \in N_{0}, K_{n+1} \subset K_{n}$. Since $K_{0} \equiv$ $S^{*}(1 / 2)$, Ruscheweyh's result implies that for each $n \in N_{0}, K_{n}$ is a subclass of $S^{*}$.

We denote by $R_{n}, n \in N_{0}$, the subclass of $A$ whose members are characterized by the condition

$$
\operatorname{Re}\left\{\frac{D^{n+1} f(z)}{D^{n} f(z)}\right\}>\frac{n}{n+1}, \quad z \in E .
$$

It follows immediately that $R_{0} \equiv S^{*}$ and that for each $n \geqslant 1, R_{n} \subset K_{n}$. Thus, for each $n \in N_{0}, R_{n}$ is a subclass of $S^{*}$. One can readily prove that $R_{n+1} \subset R_{n}$ for every $n \in N_{0}$. These inclusion relations and the fact that $z /(1-x z)$ belongs to $R_{n}$ if and only if $|x| \leqslant 1 / n$, along with a result of Ruscheweyh [3, Corollary 2, Theorem 4] imply that $\cap_{n \in N_{0}} R_{n}=\{z\}$.

The following interesting results are due to Libera [2].

TheOrem A. If $f \in S^{*}$, then so does the function $F$, defined by

$$
F(z)=\frac{2}{z} \int_{\dot{0}}^{z} f(t) d t
$$

TheOREM B. If $f \in K$, then so does the function $F$, defined by (6).

TheOrem C. If $f \in C$, then so does the function $F$, defined by (6).

In this paper, along with other things, we prove that the above-mentioned results of Libera continue to hold for much wider classes than the ones for which he has proved them.

THEOREM 1. Let $f \in A$ and for a given $n \in N_{0}$ satisfy the condition

$$
\operatorname{Re} \frac{D^{n+1} f(z)}{D^{n} f(z)}>\frac{2 n-1}{2(n+1)}, \quad z \in E
$$

Let $F$ be defined by (6). Then $F \in R_{n}$.

Proof. The condition (7) when expressed in terms of $F$ reads as follows:

$$
\operatorname{Re}\left[\frac{(n+2) D^{n+2} F(z) / D^{n+1} F(z)-n}{(n+1)-(n-1) D^{n} F(z) / D^{n+1} F(z)}\right]>\frac{2 n-1}{2(n+1)} \quad(z \in E),
$$

where we have made use of the identity

$$
z\left(D^{m} F(z)\right)^{\prime} \equiv(m+1) D^{m+1} F(z)-m D^{m} F(z) \quad \text { for every } m \in N_{0} .
$$


We have to prove that $(8)$ implies the inequality

$$
\operatorname{Re} \frac{D^{n+1} F(z)}{D^{n} F(z)}>\frac{n}{n+1} \quad(z \in E) .
$$

Define $w$ in $E$ by

$$
\begin{aligned}
G(z) & =\frac{D^{n+1} F(z)}{D^{n} F(z)}=\frac{n}{n+1}+\frac{1}{n+1} \frac{1-w(z)}{1+w(z)} \\
& =\frac{(n+1)+(n-1) w(z)}{(n+1)(1+w(z))}
\end{aligned}
$$

Evidently $w(0)=0$. Differentiating (10) logarithmically and simplifying, we obtain

$$
\begin{aligned}
& {\left[\frac{(n+2) D^{n+2} F(z) / D^{n+1} F(z)-n}{(n+1)-(n-1) D^{n} F(z) / D^{n+1} F(z)}\right]} \\
& \quad=\left[\frac{(n+1)+(n-1) w(z)}{(n+1)(1+w(z))}-\frac{1}{n+1} \cdot \frac{z w^{\prime}(z)}{w(z)} \cdot \frac{w(z)}{1+w(z)}\right] .
\end{aligned}
$$

If $\operatorname{Re} G\left(z_{0}\right)=n /(n+1)$ for a certain $z_{0}$ belonging to $E$ and $\operatorname{Re} G(z)>$ $n /(n+1)$ for $|z|<\left|z_{0}\right|$, then $|w(z)|<\left|w\left(z_{0}\right)\right|=1$ for $|z|<\left|z_{0}\right|$ and of course $w\left(z_{0}\right) \neq-1$.

Applying Jack's lemma [1] to $w(z)$ at the point $z_{0}$ and letting $z_{0} w^{\prime}\left(z_{0}\right) / w\left(z_{0}\right)$ $=k$, so that $k>1$, we obtain from (11)

$$
\begin{array}{r}
\operatorname{Re}\left[\frac{(n+2) D^{n+2} F\left(z_{0}\right) / D^{n+1} F\left(z_{0}\right)-n}{(n+1)-(n-1) D^{n} F\left(z_{0}\right) / D^{n+1} F\left(z_{0}\right)}\right] \\
=\frac{n}{n+1}-\frac{k}{2(n+1)}<\frac{2 n-1}{2(n+1)},
\end{array}
$$

which contradicts (8). This proves that $\operatorname{Re} G(z)>n /(n+1)$ in $E$ and hence $F \in R_{n}$. This completes the proof of Theorem 1 .

Putting $n=0$ and $n=1$ in Theorem 1, we obtain the following interesting results which assert that Theorems $A$ and $B$ of Libera hold under much weaker assumptions.

Corollary A. If $f$ is starlike of order $-1 / 2$, that is, if $f \in S^{*}(-1 / 2)$, then the function $F$, defined by (6), belongs to $S^{*}$.

Corollary B. If $f$ is convex of order $-1 / 2$, that is, if $f \in K(-1 / 2)$, then the function $F$, defined by (6), belongs to $K$.

It is well known that each $f \in S$ is convex in the $\operatorname{disc}|z|<2-\sqrt{3}$ and hence, from Theorem B, it follows that the Libera transforms $(2 / z) \int_{0}^{z} f(t) d t$ of members of $S$ map the disc $|z|<r_{0}, r_{0}>2-\sqrt{3}$, onto convex domains. However, in view of Corollary B, we can strengthen this result. 
Corollary $\mathrm{B}^{\prime}$. If $f \in S$, then the function $F$ defined by (6) maps $|z|<r_{1}$, $r_{1}>4-\sqrt{13}=0.394 \ldots$, onto a convex domain.

Proof. It is well known that for $f \in S$, we have

$$
\left|\frac{z f^{\prime \prime}(z)}{f^{\prime}(z)}-\frac{2 r^{2}}{1-r^{2}}\right|<\frac{4 r}{1-r^{2}} \quad(|z|=r<1),
$$

from which we deduce that

$$
\operatorname{Re}\left(1+\frac{z f^{\prime \prime}(z)}{f^{\prime}(z)}\right)>-\frac{1}{2}
$$

in $|z|<r_{1}$, where $r_{1}=4-\sqrt{13}$, is the smallest positive root of the equation $r^{2}-8 r+3=0$. Corollary $\mathrm{B}^{\prime}$ now follows from Corollary $\mathrm{B}$.

The results of Corollaries $A$ and $B$ were orally communicated to the authors by Professors St. Ruscheweyh and V. Singh.

Our next result shows that Theorem $\mathrm{C}$ of Libera holds under much weaker hypotheses.

Corollary C. Let $f \in A$ satisfy the condition

$$
\operatorname{Re}\left\{f^{\prime}(z) / g^{\prime}(z)\right\}>0 \quad(z \in E)
$$

where $g$ is any member of $K(-1 / 2)$. Then the function $F$, defined by (6), is in $C$.

Proof. Since $g \in K(-1 / 2)$, from Corollary B it follows that

$$
G(z)=\frac{2}{z} \int_{0}^{z} g(t) d t \in K
$$

From the hypothesis, we have

$$
\operatorname{Re}\left\{\frac{z F^{\prime \prime}(z)+2 F^{\prime}(z)}{z G^{\prime \prime}(z)+2 G^{\prime}(z)}\right\}=\operatorname{Re} \frac{f^{\prime}(z)}{g^{\prime}(z)}>0 \quad(z \in E) .
$$

We shall prove that $F(z)$ is close-to-convex with respect to the convex function $G(z)$, that is,

$$
\operatorname{Re} \frac{F^{\prime}(z)}{G^{\prime}(z)}>0 \quad(z \in E)
$$

To prove (14), put

$$
\frac{F^{\prime}(z)}{G^{\prime}(z)}=\frac{1-w(z)}{1+w(z)}
$$

The function $w(z)$ defined in this way is clearly regular in $E, w(0)=0$ and $w(z) \neq-1$ in $E$. The desired result would follow if we prove that $|w(z)|<1$ in E.

From (15), we at once obtain

$$
\frac{z F^{\prime \prime}(z)+2 F^{\prime}(z)}{z G^{\prime \prime}(z)+2 G^{\prime}(z)}=\frac{1-w(z)}{1+w(z)}-\frac{2 z w^{\prime}(z)}{(1+w(z))^{2}}\left(\frac{z G^{\prime \prime}(z)}{G^{\prime}(z)}+2\right)^{-1} \text {. }
$$

Let us assume that $|w(z)| \Varangle 1$ in $E$. Then, by Jack’s lemma, there exists a 
point $z_{0}$ in $E$ such that $z_{0} w^{\prime}\left(z_{0}\right)=k w\left(z_{0}\right)$, with $\left|w\left(z_{0}\right)\right|=1$ and $k>1$. Putting $z=z_{0}$ and $w\left(z_{0}\right)=e^{i \theta}, 0 \leqslant \theta \leqslant 2 \pi$, in (16), we obtain

$$
\frac{z_{0} F^{\prime \prime}\left(z_{0}\right)+2 F^{\prime}\left(z_{0}\right)}{z_{0} G^{\prime \prime}\left(z_{0}\right)+2 G^{\prime}\left(z_{0}\right)}=\frac{1-e^{i \theta}}{1+e^{i \theta}}-\frac{2 k e^{i \theta}}{\left(1+e^{i \theta}\right)^{2}}\left[\frac{z_{0} G^{\prime \prime}\left(z_{0}\right)}{G^{\prime}\left(z_{0}\right)}+2\right]^{-1}
$$

Since $\operatorname{Re}\left(1-e^{i \theta}\right) /\left(1+e^{i \theta}\right)=0, k e^{i \theta} /\left(1+e^{i \theta}\right)^{2}$ is real and positive and $\operatorname{Re}\left(\left(z G^{\prime \prime}(z) / G^{\prime}(z)\right)+1\right)>0$ in $E$, from the last relation a contradiction to our hypothesis (13) would follow. We must therefore have $|w(z)|<1$ in $E$ and Corollary $\mathrm{C}$ is established.

THEOREM 2. If $f \in R_{n}$, then the function $F$, defined by

$$
F(z)=\frac{n+1}{z^{n}} \int_{0}^{z} t^{n-1} f(t) d t
$$

belongs to $R_{n+1}$.

Proof. From the definition of F, we have

$$
\begin{aligned}
(n+1) D^{n} f(z) & =D^{n}\left(n F(z)+z F^{\prime}(z)\right) \\
& =n D^{n} F(z)+z\left(D^{n} F(z)\right)^{\prime} \\
& =(n+1) D^{n+1} F(z) \quad \text { (using (9)). }
\end{aligned}
$$

Similarly $(n+1) D^{n+1} f(z)=(n+2) D^{n+2} F(z)-D^{n+1} F(z)$. From these relations and the fact that $f \in R_{n}$, we conclude that

$$
\operatorname{Re}\left\{\frac{(n+2) D^{n+2} F(z)-D^{n+1} F(z)}{(n+1) D^{n+1} F(z)}\right\}=\operatorname{Re} \frac{D^{n+1} f(z)}{D^{n} f(z)}>\frac{n}{n+1} \quad(z \in E)
$$

from which it follows that

$$
\operatorname{Re} \frac{D^{n+2} F(z)}{D^{n+1} F(z)}>\frac{n+1}{n+2} \quad(z \in E)
$$

and therefore $F \in R_{n+1}$.

REMARK. One can show that Theorem 2 remains true on replacing $R_{n}$ by $K_{n}$ and $R_{n+1}$ by $K_{n+1}$ in the hypothesis and the conclusion, respectively.

The authors are grateful to the referee for his suggestions which greatly helped in presenting this paper in a compact form.

\section{REFERENCES}

1. I. S. Jack, Functions starlike and convex of order $\alpha$, J. London Math. Soc. (2) 3 (1971), 469-474.

2. R. J. Libera, Some classes of regular univalent functions, Proc. Amer. Math. Soc. 16 (1965), 755-758.

3. St. Ruscheweyh, New criteria for univalent functions, Proc. Amer. Math. Soc. 49 (1975), $109-115$.

Department of Mathematics, Punjabi University, Patiala, 147002, Punjab, India 